\title{
Practical Suitability of a Stand-Alone Oscillometric Central Blood Pressure Monitor: A Review of the Microlife WatchBP Office Central
}

\author{
Willem J. Verberk ${ }^{a}$ b Hao-min Cheng ${ }^{c-e} \quad$ Li-Chih Huang $^{b}$ \\ Chia-Ming Lin ${ }^{b}$ Yao-Pin Teng ${ }^{b}$ Chen-Huan Chen ${ }^{c-e}$ \\ ${ }^{a}$ Cardiovascular Research Institute Maastricht (CARIM), Maastricht University, Maastricht, \\ The Netherlands; ${ }^{\text {b}}$ Department of Research and Development, Microlife Corporation, \\ 'Department of Medical Education, Taipei Veterans General Hospital, and Departments of \\ d Medicine and e Public Health, National Yang-Ming University, Taipei, Taiwan, ROC
}

\section{Key Words}

Blood pressure $\cdot$ Central pulse pressure $\cdot$ Hypertension · Oscillometric signals · Pressure wave reflection ? Pulse volume plethysmography Pulse wave analysis

\begin{abstract}
Accumulating evidence indicates that central blood pressure (CBP) is a better cardiovascular risk predictor than brachial blood pressure (BP). Although more additional benefits of CBPbased treatment above usual hypertension treatment are to be demonstrated, the demand for implementing CBP assessment in general clinical practice is increasing. For this, the measurement procedure must be noninvasive, easy to perform, and cost- and time-efficient. Therefore, oscillometric devices with the possibility to assess CBP seem the best option. Recently, such an oscillometric BP monitor, the Microlife WatchBP Office Central, was developed, which demonstrated its high accuracy in a validation study against invasive BP measurement. Calibration errors of this device are limited because the procedure is automated, standardized, and performed at the same place of and within $30 \mathrm{~s}$ from pulse wave assessment. The transformation from the peripheral pulse wave to CBP is done by means of an individualbased pulse wave analysis according to a theory of arterial compliance and wave reflections. In addition, the device has demonstrated to enable a more reliable diagnosis of hypertension by $\mathrm{CBP}$ than by peripheral $\mathrm{BP}$, with a lower frequency of over- and underdiagnosis. Altogether, the available clinical evidence suggests that the Microlife WatchBP Office Central fulfills the criteria for general clinical use.


Verberk et al.: Practical Suitability of a Stand-Alone Oscillometric Central Blood Pressure Monitor: A Review of the Microlife WatchBP Office Central

\section{Introduction}

Noninvasive brachial or upper-arm blood pressure (BP) measurement has been the most commonly performed medical procedure for more than a century. This is because it is easily performed and has been demonstrated to be a useful cardiovascular risk predictor. However, the gold standard for BP measurement is invasive BP (IBP) measurement, and although noninvasive BP (NIBP) may correlate fairly well with IBP, values generally differ, and there can be large discrepancies on an individual level [1]. Within the arterial system, the BP in the ascending aorta, referred to as central BP (CBP), is expected to be a better cardiovascular predictor than BP at the arm (peripheral BP) because of its closer spatial relationship with the coronary and cerebral arteries. This hypothesis is supported by accumulating evidence demonstrating that CBP measurement better correlates with cardiovascular risk [2-5] and is more related to left ventricular mass [6] than peripheral BP measurement. In addition, studies have shown that CBP measurement better distinguishes the effect of antihypertensive treatment [7-10]. The suspected higher value of CBP as a cardiovascular risk predictor than brachial BP has increased the demand to implement CBP measurement as a standard procedure in the clinical environment. However, IBP by means of cardiac catheterization, which is the most direct and accurate method, cannot be used, and therefore alternatives are needed. The present paper aims at describing alternative methods that may be suitable for general clinical practice and one device in particular: the WatchBP Office Central (Microlife Corp., Taipei, Taiwan, ROC; fig. 1).

\section{Applanation Tonometry}

The first and most commonly used method for the noninvasive estimation of CBP is applanation tonometry. This method is based on the principle that the sensor applanates (flattens) the arterial wall causing the radius of the wall curvature to become infinite so that the external pressure is identical to the internal pressure and the output totally reflects the internal pressure [11]. Generally, the pressure sensor is placed over the radial or carotid artery to acquire the arterial pressure waveform (PWF). This waveform is then calibrated

Fig. 1. Microlife WatchBP Office Central, an oscillometric BP monitor that can assess CBP.

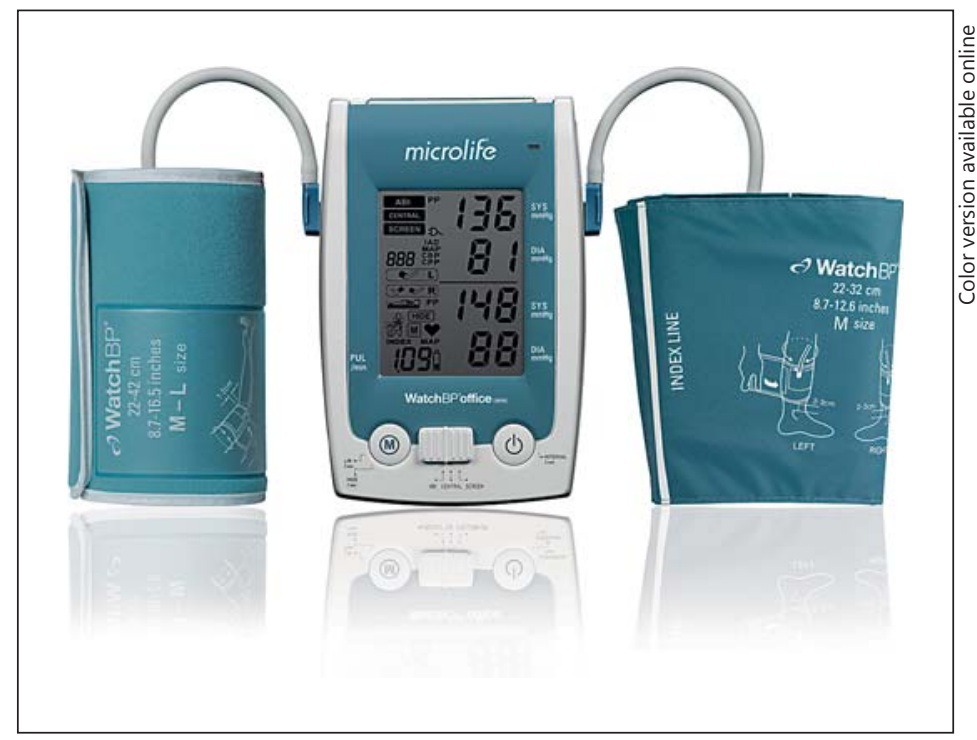


Verberk et al.: Practical Suitability of a Stand-Alone Oscillometric Central Blood

Pressure Monitor: A Review of the Microlife WatchBP Office Central

from an earlier-performed peripheral BP measurement. Subsequently, a generalized transfer function (GTF) is used to transform the radial PWF into a central PWF from which the central systolic BP (cSBP) and central pulse pressure (cPP) are assessed. Although applanation tonometry is more convenient than IBP measurement, it does not seem suitable for routine clinical practice either because it requires skills from the observer, it is time-consuming or the equipment is expensive.

In addition, applanation tonometry is significantly influenced by SBP amplification from the brachial to radial arteries so that the radial waveform is calibrated with an incorrect/ underestimated SBP resulting in consequent underestimation of cSBP $[12,13]$, a topic that is explained in more detail later in the current review.

\section{Automated Oscillometric Devices for CBP Measurement}

Currently, there are several automated oscillometric BP monitors on the market that claim to enable CBP assessment noninvasively. These devices measure the PWF of either the radial or brachial artery and use different techniques to estimate the CBP value $[14,15]$. The procedure is fully automated, does not require more efforts and only needs limited extra time than the standard oscillometric BP measurement. Some of these devices may reach the accuracy and convenience levels that are suitable for regular routine CBP measurement.

\section{WatchBP Office Central}

The WatchBP Office Central BP monitor is a validated oscillometric BP monitor $[16,17]$ that has been extended with the possibility to assess CBP. The device measures brachial SBP and diastolic BP (DBP) noninvasively as usual. However, where the cuff normally totally deflates after the peripheral BP measurement, the cuff now stops deflating at $60 \mathrm{~mm} \mathrm{Hg}$ cuff pressure (or at diastolic pressure if this is less than $60 \mathrm{~mm} \mathrm{Hg}$ ) to keep a stable pressure on the arteries for approximately $30 \mathrm{~s}$ to acquire brachial pulse volume plethysmography (pulse volume recording). During these $30 \mathrm{~s}, 20-30 \mathrm{PWF}$ are recorded and analyzed. The time that is needed to determine the CBP value may vary among patients; i.e. with faster heart rate, less time is required for collecting the number of required PWFs. In order to increase accuracy, the average PWF is determined from multiple PWFs. From the average PWF, some characteristic points (parameters) are identified that are directly related to arterial compliance and wave reflections. With these parameters, cSBP and cPP is estimated based on separate multivariate regression equations calibrated to the measured noninvasive brachial SBP and DBP. When sufficient PWFs are obtained and analyzed, the cSBP and cPP are directly calculated [18]. The DBP is not provided and can be calculated as cSBP - cPP $[19,20]$.

\section{Validation}

The Association for the Advancement of Medical Instrumentation (AAMI) validation protocol for NIBP monitors requires 85 subjects performing 3 measurements, so that 255 data points are obtained. For the invasive validation study, recruitment of $\geq 15$ subjects with a minimum of 150 paired observations and a maximum of 10 paired measurements per subject is required [21]. However, currently there are no standardized validation methods for noninvasive central BP monitors although an international task force has been convened to address this issue. Therefore, for the validation of the WatchBP Office Central the most 
Fig. 2. Parameters identified from the noninvasively brachial pulse volume plethysmography (PVP) as obtained with the WatchBP Office Central for the prediction of aortic SBP. Ad = Area under the curve during diastole; As = area under the curve during systole; $\mathrm{DBP}=$ pressure value at end-diastole; ESP = end-systolic pressure, pressure value at onset of diastole; SBP2 = pressure value of the late systolic shoulder produced by wave reflections.

Verberk et al.: Practical Suitability of a Stand-Alone Oscillometric Central Blood

Pressure Monitor: A Review of the Microlife WatchBP Office Central

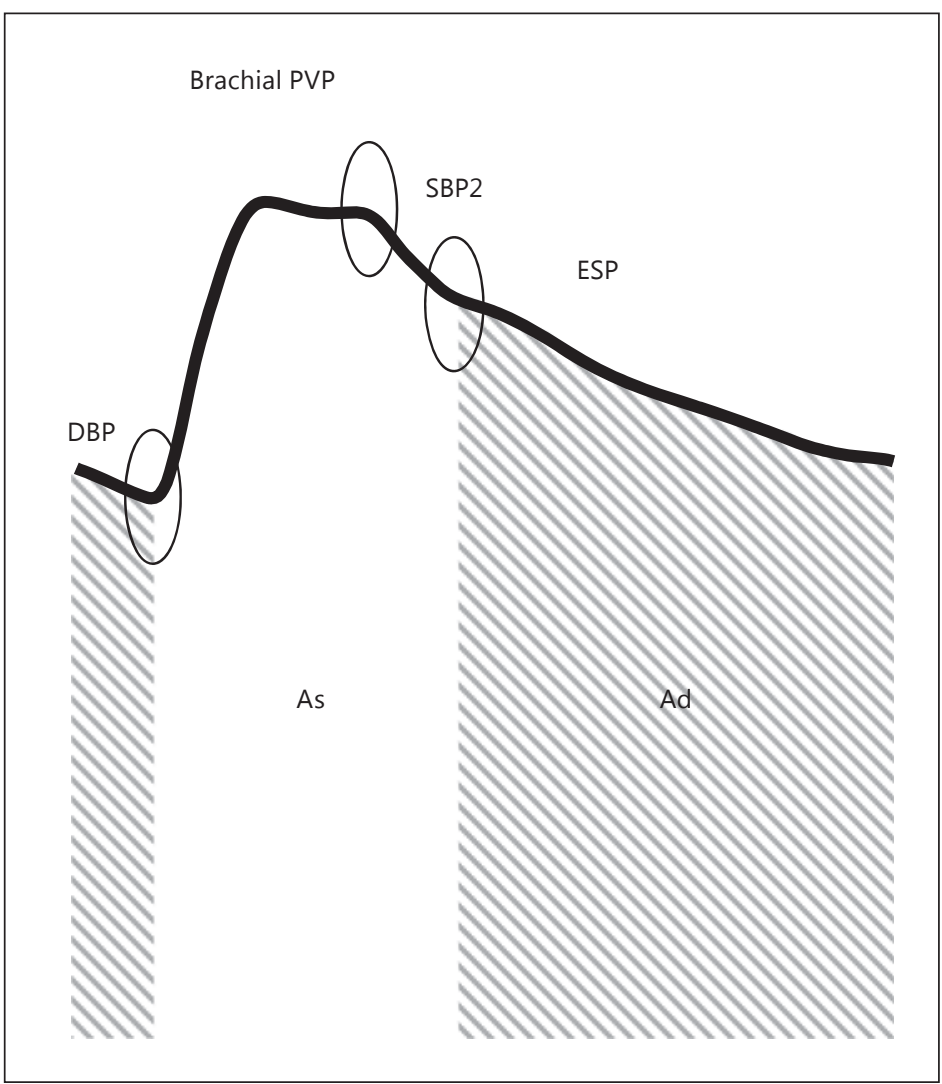

rigorous approach was chosen; 85 subjects were recruited according to the recommendations for noninvasive validation, and all requirements for the invasive reference standard were also fulfilled [20]. IBP was measured using a fluid-filled catheter which was advanced to the ascending aorta via the right radial artery and placed $2 \mathrm{~cm}$ above the aortic valve. The oscillometric measurement was performed at the left arm (patients with inter-arm differences of $3 \mathrm{~mm} \mathrm{Hg}$ or more were excluded). Results showed that the mean differences between noninvasive and invasive cSBP, cPP, and DBP (with SDs) were $-0.6 \pm 5.5,-0.4 \pm 7.0$, and -0.2 $\pm 6.5 \mathrm{~mm} \mathrm{Hg}$, respectively, well within the $5 \pm 8 \mathrm{~mm} \mathrm{Hg}$ defined by AAMI SP10 [22].

\section{Pulse Wave Analysis versus GTF}

The high accuracy of the Microlife device may be largely ascribed to a new method that is used which enables direct analysis of the PWF, herein referred to as pulse wave analysis (PWA). Figure 2 demonstrates the parameters obtained by means of brachial pulse volume plethysmography with the WatchBP Office Central that are used in a multivariate prediction model [19] as presented with the formula: predicted aortic SBP $=0.465 \times \mathrm{SBP} 2+0.465 \times \mathrm{ESP}$ $+0.490 \times$ As $-0.069 \times \mathrm{Ad}-0.753$. The parameters that are used for calculating cSBP and cPP directly are related to arterial compliance [the points at onset and end of diastole, end of systole (ESP) and the areas under the curve during systole (As) and diastole (Ad)] [23] and to the intensity of pressure wave reflection [late systolic shoulder (SBP2)] [24].

PWA is more specific for the individual patient than the method using GTF for estimating CBP [25]. The GTF is based on population average and does not take into account individual 
Verberk et al.: Practical Suitability of a Stand-Alone Oscillometric Central Blood

Pressure Monitor: A Review of the Microlife WatchBP Office Central

characteristics such as age, gender, and disease status. For this reason, significant errors in CBP estimation may exist as a consequence of individual variations [26-28]. In addition, some GTF-based devices calculate cPP by subtracting cuff DBP from cSBP because clinical evidence showed that central DBP does not significantly differ from peripheral DBP [19]. However, calculating cPP this way appears to be less accurate than the directly assessed cPP by means of PWA as the GTF-based method bears measurement errors for both cuff SBP and DBP [25].

\section{Differences between IBP and NIBP Measurement}

IBP measurement is considered to be the gold standard of BP measurement in general. Manual BP measurement assessed by a qualified observer using a mercury BP monitor is considered to be the gold standard of NIBP measurement. However, invasively measured BP values are generally not similar to NIBP values. This may sometimes lead to confusion when central (invasive) BP is noninvasively estimated.

Studies comparing IBP with NIBP using mercury devices showed that IBP is generally higher (7-25 mm Hg higher) than NIBP for SBP values [29, 30] and lower for DBP values [29]. Since mercury devices are used for validating oscillometric and auscultatory devices [31,32], the same differences with IBP measurement may be expected for these devices as for the mercury sphygmomanometers. Ribezzo et al. [33] demonstrated this in a randomized crossover trial among 50 adult patients with an average auscultatory BP value of $125 / 63 \mathrm{~mm}$ $\mathrm{Hg}$ (200 comparisons). Compared with IBP measurement, both auscultatory (aneroid) and oscillometric systolic values were significantly lower $(-9.74 \pm 13.8$ and $-10.80 \pm 14.9 \mathrm{~mm} \mathrm{Hg}$, respectively), where DBP values were significantly higher (5.13 \pm 7.1 and $3.62 \pm 6.0 \mathrm{~mm} \mathrm{Hg}$, respectively). However, systolic IBP does not always appear higher than NIBP. In a study of 87 infants (postmenstrual age ranging from 26 to 52 weeks) with very low mean BP values (from the 243 measurements, 132 were lower than $40 \mathrm{~mm} \mathrm{Hg}$ ), NIBP measurement overestimated IBP values particularly in smaller and sicker infants at lower IBP. The mean BP was $9 \mathrm{~mm}$ Hg higher for NIBP measurement than for IBP measurement. In addition, the authors concluded that NIBP measurement led to an unacceptable degree of overestimation at mean IBP of less than $40 \mathrm{~mm} \mathrm{Hg}$ [34]. These findings indicate that NIBP measurement overestimates at low IBP and underestimates at higher IBP values. In their study, Wax et al. [35] showed among 15,310 subjects that NIBP was likely to be higher than systolic IBP at lower IBPs, and NIBP was likely to be lower than IBP at higher IBPs, with crossover points estimated at 111 and $90 \mathrm{~mm} \mathrm{Hg}$ for systolic and diastolic IBP, respectively. Although the above findings might give a good overall impression about the average differences between IBP and NIBP values, individual characteristics (age, height, weight, heart rate, etc.) can also influence the difference between these values. In addition, Murray and Gorven [1] showed that the pressure contour can affect the difference as subjects exhibiting prominent inotropic pressure pulses had significantly greater differences between NIBP and IBP than patients not exhibiting them.

\section{Differences between Central and Peripheral BP Measurement}

Apart from the difference between IBP and NIBP measurement, the place of measurement is also of influence. Due to the changing vessel characteristics and degree of wave reflection, the shape of the PWF changes continuously throughout the arterial tree [36]. The PWF travels from the elastic central arteries to the stiffer peripheral arteries so that the upper portion of the wave becomes narrower, the systolic peak becomes more prominent, and systolic pressure increases [37]. In addition, the wave is a sum of a forward and a reflected wave, and because 
the wave reflection (augmentation) is more abundant at the peripheral tree, the wave and thus SBP are higher than in the ascending aorta. Consequently, radial BP is higher than brachial BP for both IBP [38] and NIBP [39] values. Although the wave reflection theory may provide a plausible description of CBP morphology, recent studies indicate that the influence of wave reflection on CBP may be less than initially assumed due to wave dispersion along the aorta and entrapment of reflected waves in the periphery. Accumulating evidence has shown that the reservoir function has a significant contribution to the augmentation of CBP [40]. The aortic reservoir represents the buffering role of the highly elastic proximal aorta, and the reservoir pressure is the minimum hydraulic force needed for the ventricle to generate the blood flow. The magnitude of aortic reservoir pressure highly depends on the arterial compliance because high compliance prevents excessive pulsatility in BP and left ventricular power expenditure. When compliance decreases, the reservoir function becomes impaired and lowers the pressure buffering capacity. This leads to augmented BP and results in left ventricular hypertrophy due to the increased myocardial load. The reservoir pressure is highly correlated with changes in proximal aortic volume, and parameters from the reservoir wave can predict cardiovascular events and correlates with left ventricular mass [40-42]. The excess pressure integral is the difference between the measured BP waveform and reservoir pressure, and is a measure of the extra work needed by the left ventricle. The excess pressure integral, which also accounts for wave reflection, has shown to indicate cardiovascular dysfunction and to be an independent predictor of cardiovascular events [42].

\section{Issues Related to Calibration}

\section{Point of Calibration}

If PWF is assessed at the radial artery but calibration for SBP is performed at the brachial artery, this could result in an underestimation of radial systolic, mean, and pulse pressure because of brachial-to-radial pressure amplification $[12,13,43]$. In order to compensate for this pressure difference, an algorithm implemented in GTF, PWA or alternatives may be needed [37, 44]. Still, thus far this calibration procedure has been a potential cause of error, which applies to both calibration based on SBP and DBP and on mean arterial pressure (MAP) and DBP. For calibration based on MAP and DBP, it is thought that there is not much change through the arterial tree [45], and therefore it may be a better choice for calibration purpose [46]. Wassertheurer and Baumann [47] demonstrated in their prospective study of the association of peripheral (brachial) SBP and cSBP and all-cause mortality that only cSBP assessed with mean and diastolic pressure calibration was significantly related to mortality. cSBP calibrated with peripheral SBP and DBP did not significantly predict mortality. A similar finding was obtained by Protogerou et al. [48] who showed that 24-hour cSBP had a significantly stronger association with left ventricular mass index when the calibration was done with mean and diastolic BP than when calibrated with systolic and diastolic pressure values. However, the use of SBP and DBP, or MAP and DBP, is likely device specific because, for example, the WatchBP Office Central might produce a large error in cSBP when using MAP and DBP for calibration [49].

\section{Short-Term BP Variability}

Calibration of most devices is done with an oscillometric device. Apart from the fact that the whole measurement depends on the accuracy of that device, the time delay between the oscillometric measurement and the pressure wave assessment may have its influence due to short-term BP variability [50], which is particularly related to aortic stiffness in hypertension [51]. This indicates that a larger time interval between calibration and PWF assessment may increase the chance of inaccurate CBP values. 
Verberk et al.: Practical Suitability of a Stand-Alone Oscillometric Central Blood

Pressure Monitor: A Review of the Microlife WatchBP Office Central

\section{BP Monitor Used for Calibration}

If calibration is performed with a BP monitor separated from the device that measures the PWFs, the calibration device significantly influences the CBP value. Not only different types of calibration devices (aneroid or oscillometric) [52] but also different validated oscillometric monitors can lead to different results as each manufacturer has its own algorithm. In order to pass the AAMI protocol, the mean difference from the reference (mercury sphygmomanometer) must be $5 \mathrm{~mm} \mathrm{Hg}$ or less [21]. In theory, this means that two validated oscillometric devices could still differ on average $10 \mathrm{~mm} \mathrm{Hg}$ from each other. In addition, validated devices from the same manufacturer demonstrated inter-device differences of almost $4 \mathrm{~mm}$ $\mathrm{Hg}$ for SBP [53] and more than $3 \mathrm{~mm} \mathrm{Hg}$ for DBP [54].

Overall, calibration is prone to error $[26,45,55]$, which suggests consensus is needed regarding the procedure as different methods and/or equipment may lead to different CBP values [56].

\section{Higher Central than Peripheral BP Values}

Despite the fact that invasive peripheral BP is higher than CBP due to pressure amplification, sometimes oscillometric CBP monitors, such as the WatchBP Office Central, may present lower peripheral than central BP values. Although this seems to be a contradiction, it makes sense if one has a full understanding of the device and different BP measurement methods. As previously described, cuff-based peripheral NIBP is not the same as IBP. The first one is based on and validated with mercury sphygmomanometers that underestimate systolic intra-arterial BP values higher than approximately $110 \mathrm{~mm} \mathrm{Hg}$ [35], and this can easily lead to a difference of more than $10 \mathrm{~mm} \mathrm{Hg}$ [29]. Since the degree of systolic pressure amplification depends on a number of variables, some people have less pulse wave amplification, e.g. subjects of short stature or those with a low heart rate, and thus a smaller difference between peripheral and central BP [37]. If the difference between IBP and NIBP is greater than the difference caused by amplification from central to peripheral arteries, then the device shows a lower peripheral BP than the central BP value.

\section{Sphygmocor: The Gold Standard?}

Most studies of CBP measurement are performed by means of applanation tonometry using a Sphygmocor device. For this reason, the device is often referred to as the gold standard for noninvasive CBP assessment or used as a 'validation' tool [57, 58]. However, because of earlier-described problems related to NIBP calibration and influence of observer bias [59], it does not fulfil the requirements for validation, leaving IBP measurement as the only option for proper validation. This means that, according to a recognized protocol, oscillometric CBP monitors that are not compared against IBP measurement cannot be considered validated.

\section{Use in Clinical Practice}

\section{CBP-Based Treatment}

Currently, 2 randomized clinical trials have demonstrated the usefulness of treatment based on CBP measurement and augmentation index as compared to conventional therapy $[60,61]$. Borlaug et al. [60] demonstrated among 50 patients (23 intervention and 27 control patients) with heart failure that, after 6 months, subjects who were randomized to active treatment were more likely to receive additional vasoactive therapy leading to greater improvement in peak oxygen consumption as compared with controls $(1.37 \pm 3.76$ vs. -0.65 
Verberk et al.: Practical Suitability of a Stand-Alone Oscillometric Central Blood Pressure Monitor: A Review of the Microlife WatchBP Office Central

$\pm 2.21 \mathrm{ml} \mathrm{min}^{-1} \mathrm{~kg}^{-1}, \mathrm{p}=0.025$ ), with similar reductions in aortic augmentation. Sharman et al. [61] showed among 286 patients that CBP-based hypertension management resulted in significantly less medication needed to achieve BP control than usual care without adverse effects on left ventricular mass, aortic stiffness, or quality of life.

The different results of these studies are highly related to the way usual care was performed: in the study by Sharman et al. [61], treatment was based on a variety of tests (office BP, 7-day home BP, 24-hour ambulatory BP, two-dimensional left ventricular mass index, symptoms, and other clinical considerations as per absolute cardiovascular risk), whereas Borlaug et al. [60] relied solely on office BP measurement. In addition, there was a difference in cut-off values for CBP measurement: Sharman et al. [61] used age- and sexspecific normative cSBP values derived from a large population study [62] and defined the normative range as within +1 SD from the mean normative values. Borlaug et al. [60] aimed at reducing the augmentation index to $0 \%$ with maintenance of cSBP in the range of 85-100 $\mathrm{mm} \mathrm{Hg}$. The above examples illustrate the need for standardized threshold values for CBP.

\section{Normal Values}

For use in routine general practice, clinicians need guidance on how to interpret the measured CBP values. For this, a threshold definition is a first requirement. Although still under debate, Cheng et al. [63] presented a promising proposal. Based on their study with a derivation cohort of 1,272 individuals and a median follow-up of 15 years using the relationship of CBP and cardiovascular mortality as an outcome, the authors came to systolic/ diastolic threshold values of 110/80 mm Hg for optimal BP and 130/90 mm Hg for hypertension, respectively. Subsequently, the derived thresholds were tested in a validation cohort of 2,501 individuals with a median follow-up of 10 years. Compared to optimal BP, the risk of cardiovascular mortality increased significantly in subjects with hypertension (hazard ratio: 3.08, 95\% confidence interval: 1.05-9.05) [63].

The Microlife WatchBP Office Central was investigated for its diagnostic accuracy, i.e. the ability to detect hypertension, in clinical practice using the proposed threshold values [64]. In 138 subjects (aged 30-93 years) without previous use of antihypertensives and a hypertension prevalence of $52 \%$ (invasive CBP $\geq 130 / 90 \mathrm{~mm} \mathrm{Hg}$ ), simultaneous automatic pressure readings were performed with the cuff of the CBP monitor placed on the left arm and an arterial catheter advanced into the ascending aorta at $2 \mathrm{~cm}$ above the aortic valve through the right radial artery. The reference hypertension (yes/no) was based on IBP measurement (threshold: $\geq 130 / 90 \mathrm{~mm} \mathrm{Hg}$ for hypertension) which was compared with noninvasive peripheral BP measurement (threshold 140/90 mm Hg) and noninvasive CBP measurement (threshold: 130/90 mm Hg) using the WatchBP Office Central. The accuracy of diagnosing hypertension based on noninvasive peripheral BP measurement showed a sensitivity of $49 \%$ and a specificity of $94 \%$, whereas for noninvasively measured CBP, the sensitivity and specificity values were 93 and 95\%. The authors concluded that, under the assumption that direct IBP measurement in the aorta (invasive CBP) is the best cardiovascular risk predictor, peripheral NIBP often leads to underestimation of invasive CBP and therefore fails to detect high BP, which could be prevented by using noninvasive CBP estimation [64].

\section{Cost-Effectiveness of CBP Monitoring}

Another part that is required before noninvasive CBP assessment could be implemented in routine healthcare is the cost-effectiveness of using this new method for diagnosing hypertension. Recently, lifetime costs and quality-adjusted life years (QALYs) were estimated for CBP and peripheral BP monitoring using a Markov cohort model [65]. Model calibration and probabilistic sensitivity analysis on primary care representatives, in 10-year age brackets, from age 35 to over 75 years of age showed that CBP-based hypertension management was 
Table 1. Advantages of the Microlife WatchBP Office Central for CBP assessment

Validated against IBP measurement

The calibration method is fully automated, standardized and implemented in the BP monitor

Pulse wave assessment is performed within $30 \mathrm{~s}$ after calibration

Calibration is performed in the same place the pulse wave form is assessed

Tested for diagnosis of hypertension based on the IBP value

Limited operator skills required

Limited extra time required as compared to regular BP measurement

The PWA algorithm is based on the cardiovascular risk related to the parameters of the individual patient

$\mathrm{CBP}=$ Central $\mathrm{BP} ; \mathrm{IBP}=$ invasive $\mathrm{BP} ; \mathrm{PWA}=$ pulse wave analysis.

more cost-effective than management based on peripheral BP for men and women across all age subgroups. Mean incremental cost-effectiveness ratios ranged from GBP 226 to 2,750 (USD 350-4,250; conversion rate October 2015) per QALY gained, which is far below the threshold of e.g. GBP 20,000 (USD 30,930) per QALY gained according to NICE. This suggests that the long-term benefits of improved diagnostic performance justify the cost increase. However, more research on this topic is needed as e.g. the additional benefits could be mainly related to more intensified treatment so that the same results might be obtained when using a lower peripheral BP threshold value for hypertension management.

\section{Conclusion}

According to the available evidence, the Microlife WatchBP Office Central seems potentially well-suited to use in the primary care setting (table 1). The measurement procedure does not take much more time than regular BP measurement and is less operator dependent than handheld tonometry. The device is highly accurate as demonstrated in an extended validation study, and the procedure may be cost-effective. The reason for its accuracy may be largely ascribed to the automated standardized calibration procedure which is performed with the same device that assesses the PWF and takes place within $30 \mathrm{~s}$ from PWF assessment at the same artery. This decreases the chance of errors due to inter-device differences, shortterm BP variability and pulse wave amplification. The automated standardized calibration procedure might not demonstrate its advantage during clinical validations, in particular, but probably will be more relevant when used by many healthcare professionals during routine clinical practice. The transformation from peripheral to central PWF is performed by means of PWA according to a theory of arterial compliance and wave reflections to calculate cSBP and cPP directly, which may be more reliable than GTF, at least for cPP. Finally, the device has shown to allow the diagnosis of hypertension based on IBP values. Altogether, the available evidence suggests that the WatchBP Office Central is an easy to use oscillometric device that can provide a convenient and reliable CBP estimation.

\section{Disclosure Statement}

Willem J. Verberk is an employee of Microlife Corporation Taiwan; Hao-min Cheng is an inventor of the noninvasive CBP technique. Li-Chih Huang is an employee of Microlife Corporation Taiwan; Chia-Ming Lin is an employee of Microlife Corporation Taiwan; Yao-Pin Teng is an employee of Microlife Corporation Taiwan; ChenHuan Chen is an inventor of the noninvasive CBP technique and is affiliated with National Yang-Ming University. Microlife and National Yang-Ming University have signed a contract for the transfer of the noninvasive CBP technique. The contract of technology transfer includes research funding for conducting the validation study. 
Verberk et al.: Practical Suitability of a Stand-Alone Oscillometric Central Blood

Pressure Monitor: A Review of the Microlife WatchBP Office Central

\section{References}

1 Murray WB, Gorven AM: Invasive vs non-invasive blood pressure measurements - the influence of the pressure contour. S Afr Med J 1991;79:134-139.

2 Huang CM, Wang KL, Cheng HM, Chuang SY, Sung SH, Yu WC, Ting CT, Lakatta EG, Yin FC, Chou P, Chen CH: Central versus ambulatory blood pressure in the prediction of all-cause and cardiovascular mortalities. J Hypertens 2011;29:454-459.

3 Roman MJ, Devereux RB, Kizer JR, Lee ET, Galloway JM, Ali T, Umans JG, Howard BV: Central pressure more strongly relates to vascular disease and outcome than does brachial pressure: the Strong Heart Study. Hypertension 2007;50:197-203.

4 Pini R, Cavallini MC, Palmieri V, Marchionni N, Di Bari M, Devereux RB, Masotti G, Roman MJ: Central but not brachial blood pressure predicts cardiovascular events in an unselected geriatric population: the ICARe Dicomano Study. J Am Coll Cardiol 2008;51:2432-2439.

5 Safar ME, Blacher J, Pannier B, Guerin AP, Marchais SJ, Guyonvarc'h PM, London GM: Central pulse pressure and mortality in end-stage renal disease. Hypertension 2002;39:735-738.

6 Sharman J, Stowasser M, Fassett R, Marwick T, Franklin S: Central blood pressure measurement may improve risk stratification. J Hum Hypertens 2008;22:838-844.

7 Boutouyrie P, Achouba A, Trunet P, Laurent S: Amlodipine-valsartan combination decreases central systolic blood pressure more effectively than the amlodipine-atenolol combination: the EXPLOR study. Hypertension 2010;55:1314-1322.

8 Morgan T, Lauri J, Bertram D, Anderson A: Effect of different antihypertensive drug classes on central aortic pressure. Am J Hypertens 2004;17:118-123.

9 Williams B, Lacy PS, Thom SM, Cruickshank K, Stanton A, Collier D, Hughes AD, Thurston H, O'Rourke M: Differential impact of blood pressure-lowering drugs on central aortic pressure and clinical outcomes: principal results of the Conduit Artery Function Evaluation (CAFE) study. Circulation 2006;113:1213-1225.

10 Protogerou AD, Papaioannou TG, Lekakis JP, Blacher J, Safar ME: The effect of antihypertensive drugs on central blood pressure beyond peripheral blood pressure. I. (Patho)-physiology, rationale and perspective on pulse pressure amplification. Curr Pharm Des 2009;15:267-271.

11 Miyashita H: Clinical assessment of central blood pressure. Curr Hypertens Rev 2012;8:80-90.

12 Picone DS, Climie RED, Ahuja KDK, Keske MA, Sharman JE: Brachial-to-radial SBP amplification: implications of age and estimated central blood pressure from radial tonometry. J Hypertens 2015;33:1876-1883.

13 Climie RE, Picone DS, Keske MA, Sharman JE: Brachial-to-radial systolic blood pressure amplification in patients with type 2 diabetes mellitus. J Hum Hypertens 2015, Epub ahead of print.

14 Shih YT, Cheng HM, Sung SH, Chuang SY, Hu WC, Chen CH: Is noninvasive brachial systolic blood pressure an accurate estimate of central aortic systolic blood pressure? Am J Hypertens 2015, Epub ahead of print.

15 Millasseau S, Agnoletti D: Non-invasive estimation of aortic blood pressures: a close look at current devices and methods. Curr Pharm Des 2015;21:709-718.

16 Stergiou GS, Lin CW, Lin CM, Chang SL, Protogerou AD, Tzamouranis D, Nasothimiou E, Tan TM: Automated device that complies with current guidelines for office blood pressure measurement: design and pilot application study of the Microlife WatchBP Office device. Blood Press Monit 2008;13:231-235.

17 Saladini F, Benetti E, Masiero S, Palatini P: Accuracy of Microlife WatchBP Office ABI monitor assessed according to the 2002 European Society of Hypertension protocol and the British Hypertension Society protocol. Blood Press Monit 2011;16:258-261.

18 Sung SH, Cheng HM, Chuang SY, Shih YT, Wang KL, Chen YH, Lin SJ, Yu WC, Chen CH: Measurement of central systolic blood pressure by pulse volume plethysmography with a noninvasive blood pressure monitor. Am J Hypertens 2012;25:542-548.

19 Cheng HM, Wang KL, Chen YH, Lin SJ, Chen LC, Sung SH, Ding PY, Yu WC, Chen JW, Chen CH: Estimation of central systolic blood pressure using an oscillometric blood pressure monitor. Hypertens Res 2010;33:592599.

20 Cheng HM, Sung SH, Shih YT, Chuang SY, Yu WC, Chen CH: Measurement accuracy of a stand-alone oscillometric central blood pressure monitor: a validation report for Microlife WatchBP Office Central. Am J Hypertens 2013;26:42-50.

21 Alpert BS, Quinn DE, Friedman BA: A review of the latest guidelines for NIBP device validation. Blood Press Monit 2013;18:297-302.

22 Association for the Advancement of Medical Instrumentation: American National Standard: Electronic or Automated Sphygmomanometers. Arlington, Association for the Advancement of Medical Instrumentation, 2009.

23 Liu Z, Brin KP, Yin FC: Estimation of total arterial compliance: an improved method and evaluation of current methods. Am J Physiol 1986;251:H588-H600.

24 Lin MM, Cheng HM, Sung SH, Liao CF, Chen YH, Huang PH, Chen CH: Estimation of central aortic systolic pressure from the second systolic peak of the peripheral upper limb pulse depends on central aortic pressure waveform morphology. J Hypertens 2012;30:581-586.

25 Cheng HM, Sung SH, Shih YT, Chuang SY, Yu WC, Chen CH: Measurement of central aortic pulse pressure: noninvasive brachial cuff-based estimation by a transfer function vs. a novel pulse wave analysis method. Am J Hypertens 2012;25:1162-1169. 
Verberk et al.: Practical Suitability of a Stand-Alone Oscillometric Central Blood

Pressure Monitor: A Review of the Microlife WatchBP Office Central

26 Shih YT, Cheng HM, Sung SH, Hu WC, Chen CH: Quantification of the calibration error in the transfer functionderived central aortic blood pressures. Am J Hypertens 2011;24:1312-1317.

27 Davies JI, Band MM, Pringle S, Ogston S, Struthers AD: Peripheral blood pressure measurement is as good as applanation tonometry at predicting ascending aortic blood pressure. J Hypertens 2003;21:571-576.

28 Cloud GC, Rajkumar C, Kooner J, Cooke J, Bulpitt CJ: Estimation of central aortic pressure by SphygmoCor requires intra-arterial peripheral pressures. Clin Sci (Lond) 2003;105:219-225.

29 Hunyor SN, Flynn JM, Cochineas C: Comparison of performance of various sphygmomanometers with intraarterial blood-pressure readings. BMJ 1978;2:159-162.

30 Holland WW, Humerfelt S: Measurement of blood-pressure: comparison of intra-arterial and cuff values. BMJ 1964;2:1241-1243.

31 O’Brien E, Atkins N, Stergiou G, Karpettas N, Parati G, Asmar R, Imai Y, Wang J, Mengden T, Shennan A; Working Group on Blood Pressure Monitoring of the European Society of Hypertension: European Society of Hypertension International Protocol revision 2010 for the validation of blood pressure measuring devices in adults. Blood Press Monit 2010;15:23-38.

32 O'Brien E, Petrie J, Littler W, de Swiet M, Padfield PL, Altman DG, Bland M, Coats A, Atkins N: An outline of the revised British Hypertension Society protocol for the evaluation of blood pressure measuring devices. J Hypertens 1993;11:677-679.

33 Ribezzo S, Spina E, Di Bartolomeo S, Sanson G: Noninvasive techniques for blood pressure measurement are not a reliable alternative to direct measurement: a randomized crossover trial in ICU. ScientificWorldJournal 2014;2014:353628.

34 Dasnadi S, Aliaga S, Laughon M, Warner DD, Price WA: Factors influencing the accuracy of noninvasive blood pressure measurements in NICU infants. Am J Perinatol 2015;32:639-644.

35 Wax DB, Lin HM, Leibowitz AB: Invasive and concomitant noninvasive intraoperative blood pressure monitoring: observed differences in measurements and associated therapeutic interventions. Anesthesiology 2011;115:973-978.

36 VanBeck JO, White RD, Abenstein JP, Mullany CJ, Orszulak TA: Comparison of axillary artery or brachial artery pressure with aortic pressure after cardiopulmonary bypass using a long radial artery catheter. J Cardiothorac Vasc Anesth 1993;7:312-315.

37 McEniery CM, Cockcroft JR, Roman MJ, Franklin SS, Wilkinson IB: Central blood pressure: current evidence and clinical importance. Eur Heart J 2014;35:1719-1725.

38 Bazaral MG, Welch M, Golding LA, Badhwar K: Comparison of brachial and radial arterial pressure monitoring in patients undergoing coronary artery bypass surgery. Anesthesiology 1990;73:38-45.

39 Li W-y, Wang X-h, Lu L-c, Li H: Discrepancy of blood pressure between the brachial artery and radial artery. World J Emerg Med 2013;4:294-297.

40 Schultz MG, Davies JE, Hardikar A, Pitt S, Moraldo M, Dhutia N, Hughes AD, Sharman JE: Aortic reservoir pressure corresponds to cyclic changes in aortic volume physiological validation in humans. Arterioscler Thromb Vasc Biol 2014;34:1597-1603.

41 Hametner B, Wassertheurer S, Hughes AD, Parker KH, Weber T, Eber B: Reservoir and excess pressures predict cardiovascular events in high-risk patients. Int J Cardiol 2014;171:31-36.

42 Davies JE, Lacy P, Tillin T, Collier D, Cruickshank JK, Francis DP, Malaweera A, Mayet J, Stanton A, Williams B, Parker KH, Thom SAM, Hughes AD: Excess pressure integral predicts cardiovascular events independent of other risk factors in the conduit artery functional evaluation substudy of Anglo-Scandinavian Cardiac Outcomes Trial. Hypertension 2014;64:60-68.

43 Verbeke F, Segers P, Heireman S, Vanholder R, Verdonck P, Van Bortel LM: Noninvasive assessment of local pulse pressure: importance of brachial-to-radial pressure amplification. Hypertension 2005;46:244-248.

44 Mitchell GF: Central pressure should not be used in clinical practice. Artery Res 2015;9:8-13.

45 Laugesen E, Rossen NB, Peters CD, Maeng M, Ebbehoj E, Knudsen ST, Hansen KW, Botker HE, Poulsen PL: Assessment of central blood pressure in patients with type 2 diabetes: a comparison between SphygmoCor and invasively measured values. Am J Hypertens 2014;27:169-176.

46 Soender TK, Van Bortel LM, Moller JE, Lambrechtsen J, Hangaard J, Egstrup K: Impact of calibration on estimates of central blood pressures. J Hum Hypertens 2012;26:706-710.

47 Wassertheurer S, Baumann M: Assessment of systolic aortic pressure and its association to all cause mortality critically depends on waveform calibration. J Hypertens 2015;33:1884-1889.

48 Protogerou AD, Argyris AA, Papaioannou TG, Kollias GE, Konstantonis GD, Nasothimiou E, Achimastos A Blacher J, Safar ME, Sfikakis PP: Left-ventricular hypertrophy is associated better with 24-h aortic pressure than 24-h brachial pressure in hypertensive patients: the SAFAR study. J Hypertens 2014;32:1805-1814.

49 Shih YT, Cheng HM, Sung SH, Hu WC, Chen CH: Comparison of two generalized transfer functions for measuring central systolic blood pressure by an oscillometric blood pressure monitor. J Hum Hypertens 2013;27:204210.

50 Dawson SL, Manktelow BN, Robinson TG, Panerai RB, Potter JF: Which parameters of beat-to-beat blood pressure and variability best predict early outcome after acute ischemic stroke? Stroke 2000;31:463-468.

51 Schillaci G, Bilo G, Pucci G, Laurent S, Macquin-Mavier I, Boutouyrie P, Battista F, Settimi L, Desamericq G, Dolbeau G, Faini A, Salvi P, Mannarino E, Parati G: Relationship between short-term blood pressure variability and large-artery stiffness in human hypertension: findings from 2 large databases. Hypertension 2012;60: 369-377. 
Verberk et al.: Practical Suitability of a Stand-Alone Oscillometric Central Blood

Pressure Monitor: A Review of the Microlife WatchBP Office Central

52 Amoore JN, Lemesre Y, Murray IC, Mieke S, King ST, Smith FE, Murray A: Automatic blood pressure measurement: the oscillometric waveform shape is a potential contributor to differences between oscillometric and auscultatory pressure measurements. J Hypertens 2008;26:35-43.

53 Topouchian J, Agnoletti D, Blacher J, Youssef A, Ibanez I, Khabouth J, Khawaja S, Beaino L, Asmar R: Validation of four automatic devices for self-measurement of blood pressure according to the international protocol of the European Society of Hypertension. Vasc Health Risk Manag 2011;7:709-717.

54 Takahashi H, Yoshika M, Yokoi T: Validation of Omron RS8, RS6, and RS3 home blood pressure monitoring devices, in accordance with the European Society of Hypertension International Protocol revision 2010. Vasc Health Risk Manag 2013;9:265-272.

55 Rezai MR, Goudot G, Winters C, Finn JD, Wu FC, Cruickshank JK: Calibration mode influences central blood pressure differences between SphygmoCor and two newer devices, the Arteriograph and Omron HEM-9000. Hypertens Res 2011;34:1046-1051.

56 Segers P, Mahieu D, Rietzschel ER, De Buyzere ML, Van Bortel LM: Impact of radial artery pressure waveform calibration on estimated central pressure using a transfer function approach. Hypertension 2008;52:e24-e25; author reply e26.

57 Papaioannou TG, Protogerou A, Stefanadis C: Comparison between Mobil-O-Graph and the SphygmoCor device for central systolic blood pressure estimation: consensus is required for 'validation protocols'. Blood Press Monit 2012;17:259-260; author reply 260-261.

58 Lehmann ED: Cited 'validation' references for the SphygmoCor device. Kidney Int 2001;59:1597-1598.

59 O’Rourke MF, Pauca A, Jiang XJ: Pulse wave analysis. Br J Clin Pharmacol 2001;51:507-522.

60 Borlaug BA, Olson TP, Mohamed SA, Melenovsky V, Sorrell VL, Noonan K, Lin G, Redfield MM: A randomized pilot study of aortic waveform guided therapy in chronic heart failure. J Am Heart Assoc 2014;3:e000745.

61 Sharman JE, Marwick TH, Gilroy D, Otahal P, Abhayaratna WP, Stowasser M; Value of Central Blood Pressure for GUIDing ManagEment of Hypertension Study Investigators: Randomized trial of guiding hypertension management using central aortic blood pressure compared with best-practice care principal findings of the BP GUIDE Study. Hypertension 2013;62:1138-1145.

62 McEniery CM, Yasmin, Hall IR, Qasem A, Wilkinson IB, Cockcroft JR; ACCT Investigators: Normal vascular aging: differential effects on wave reflection and aortic pulse wave velocity - The Anglo-Cardiff Collaborative Trial (ACCT). J Am Coll Cardiol 2005;46:1753-1760.

63 Cheng HM, Chuang SY, Sung SH, Yu WC, Pearson A, Lakatta EG, Pan WH, Chen CH: Derivation and validation of diagnostic thresholds for central blood pressure measurements based on long-term cardiovascular risks. J Am Coll Cardiol 2013;62:1780-1787.

64 Cheng HM, Sung SH, Chuang SY, Pearson A, Tufanaru C, White S, Yu WC, Chen CH: Diagnostic performance of a stand-alone central blood pressure monitor: application of central blood pressure in the diagnosis of high blood pressure. Am J Hypertens 2014;27:382-391.

65 Cheng HM, Pearson A, Sung SH, Yu WC, Chen CH, Karnon J: Cost-effectiveness of noninvasive central blood pressure monitoring in the diagnosis of hypertension. Am J Hypertens 2015;28:604-614. 